\title{
On Singular Integrals with Cauchy Kernel on Weight Subspaces: The Basicity Property of Sines and Cosines Systems in Weight Spaces
}

\author{
Tofig Isa Najafov ${ }^{\mathbf{1}}$ and Saeed Farahani ${ }^{\mathbf{2}}$ \\ ${ }^{1}$ Nakhchivan State University, University Campus, AZ 7000 Nakhchivan, Azerbaijan \\ ${ }^{2}$ Institute of Mathematics and Mechanics, Azerbaijan National Academy of Sciences, \\ AZ 1141 Baku, Azerbaijan \\ Correspondence should be addressed to Tofig Isa Najafov, s_sadigova@mail.ru \\ Received 3 February 2011; Accepted 28 March 2011 \\ Academic Editor: Shyam Kalla
}

Copyright (C) 2011 T. I. Najafov and S. Farahani. This is an open access article distributed under the Creative Commons Attribution License, which permits unrestricted use, distribution, and reproduction in any medium, provided the original work is properly cited.

A singular operator with Cauchy kernel on the subspaces of weight Lebesgue space is considered. A sufficient condition for a bounded action of this operator from a subspace to another subspace of weight Lebesgue space of functions is found. These conditions are not identical with Muckenhoupt conditions. Moreover, the completeness, minimality, and basicity of sines and cosines systems are considered.

\section{Introduction}

Consider the following singular operator with Cauchy kernel:

$$
[S f](t)=\frac{1}{2 \pi i} \int_{-\pi}^{\pi} \frac{f(s) d s}{1-e^{-i(s-t)}}
$$

where $f \in L_{p, \rho}(-\pi, \pi), 1<p<+\infty$, is an appropriate density, $\rho(t)$ is a weight function of the form

$$
\rho(t)=\prod_{k=1}^{r}\left|t-t_{k}\right|^{p \beta_{k}}
$$

$\left\{t_{k}\right\}_{1}^{r} \subset[-\pi, \pi]\left(t_{i} \neq t_{j}\right.$ for $\left.i \neq j\right),\left\{\beta_{k}\right\}_{1}^{r} \subset R$ are real numbers. 
Under $L_{p, \rho}$ we understand a Lebesgue weight space with the norm

$$
\|f\|_{p, \rho} \equiv\left(\int_{-\pi}^{\pi}|f(t)|^{p} \rho(t) d t\right)^{1 / p}
$$

Bounded action of the operator $S$ in the spaces $L_{p, \rho}$ plays an important role in many problems of mathematics including the theory of bases. This direction has been well developed and treated in the known monographs. We will need the following.

Statement 1. The operator $S$ is bounded in $L_{p, \rho}$ if and only if the inequalities

$$
-\frac{1}{p}<\beta_{k}<\frac{1}{q}, \quad k=\overline{1, r}, \quad \frac{1}{p}+\frac{1}{q}=1
$$

are fulfilled.

Concerning this fact a one can see the monograph [1] and papers [2-4]. Inequalities (1.4) are Muckenhoupt condition with respect to the weight function $\rho(t)$ with degrees $p \beta_{k}$. It is known that the classic system of exponents $\left\{e^{i n t}\right\}_{n \in Z}$ ( $Z$ are integers) forms a basis in $L_{p, \rho}$ if and only if inequalities (1.4) hold (see, e.g., $[3,4]$ ).

It turns that if you consider the singular operator acting on the subspace of the weighted Lebesgue space, then inequality (1.4) is not necessary for the bounded action. At different points of degeneration the change interval of the corresponding exponent is expanded. This paper is devoted to studying these issues.

\section{Some Necessary Facts}

Let $L_{p, \omega}^{0} \equiv L_{p, \omega}(0, \pi), \omega(t)$-a weight function of the form

$$
\omega(t) \equiv \prod_{k=0}^{r}\left|t-\tau_{k}\right|^{p \alpha_{k}}
$$

where $0=\tau_{0}<\tau_{1}<\cdots<\tau_{r}=\pi,\left\{\alpha_{k}\right\}_{0}^{r} \subset R$.

Denote the space of even (odd) functions in $L_{p, \rho}$ by $L_{p, \rho}^{+}\left(L_{p, \rho}^{-}\right)$, that is,

$$
L_{p, \rho}^{ \pm} \equiv\left\{f \in L_{p, \rho}: f(-t)= \pm f(t), t \in[-\pi, \pi]\right\}
$$

We'll need the following identity:

$$
\frac{1}{1-e^{i(\theta-\varphi)}}-\frac{1}{1-e^{i(\theta+\varphi)}}=\frac{i}{2} \frac{\sin \varphi}{\sin ((\varphi-\theta) / 2) \sin ((\theta+\varphi) / 2)} .
$$


Indeed, we have

$$
\begin{aligned}
& \frac{1}{1-e^{i(\theta-\varphi)}}-\frac{1}{1-e^{i(\theta+\varphi)}}=\frac{1-e^{-i(\theta-\varphi)}}{\left(1-e^{i(\theta-\varphi)}\right)\left(1-e^{-i(\theta-\varphi)}\right)}-\frac{1-e^{-i(\theta+\varphi)}}{\left(1-e^{i(\theta+\varphi)}\right)\left(1-e^{-i(\theta+\varphi)}\right)} \\
& =\frac{1-\cos (\theta-\varphi)+i \sin (\theta-\varphi)}{(1-\cos (\theta-\varphi))^{2}+\sin ^{2}(\theta-\varphi)}-\frac{1-\cos (\theta+\varphi)+i \sin (\theta+\varphi)}{(1-\cos (\theta+\varphi))^{2}+\sin ^{2}(\theta+\varphi)} \\
& =i\left[\frac{\sin (\theta-\varphi)}{2-2 \cos (\theta-\varphi)}-\frac{\sin (\theta+\varphi)}{2-2 \cos (\theta+\varphi)}\right] \\
& =i\left[\frac{\cos ((\theta-\varphi) / 2)}{2 \sin ((\theta-\varphi) / 2)}-\frac{\cos ((\theta+\varphi) / 2)}{2 \sin ((\theta+\varphi) / 2)}\right] \\
& =\frac{i}{2} \frac{\cos ((\theta-\varphi) / 2) \sin ((\theta+\varphi) / 2)-\cos ((\theta+\varphi) / 2) \sin ((\theta-\varphi) / 2)}{\sin ((\theta-\varphi) / 2) \sin ((\theta+\varphi) / 2)} \\
& =\frac{i}{2} \frac{\sin \varphi}{\sin ((\theta-\varphi) / 2) \sin ((\theta+\varphi) / 2)} .
\end{aligned}
$$

For compactness of the notation, we assume $e(x) \equiv 1 /\left(1-e^{i x}\right)$. Thus,

$$
e(\theta-\varphi)-e(\theta+\varphi)=\frac{i}{2} \frac{\sin \varphi}{\sin ((\theta-\varphi) / 2) \sin ((\theta+\varphi) / 2)} .
$$

From this identity, we can easily get the following relations:

$$
\begin{aligned}
& e(\theta-\varphi)-e(\theta+\varphi)=-\frac{i}{2} \frac{\cos (\varphi / 2)}{\cos (\theta / 2)}\left[\frac{1}{\sin ((\varphi-\theta) / 2)}+\frac{1}{\sin ((\varphi+\theta) / 2)}\right] \\
& e(\theta-\varphi)-e(\theta+\varphi)=-\frac{i}{2} \frac{\sin (\varphi / 2)}{\sin (\theta / 2)}\left[\frac{1}{\sin ((\varphi-\theta) / 2)}-\frac{1}{\sin ((\varphi+\theta) / 2)}\right] .
\end{aligned}
$$

The authors of the papers [4-7] used these relations earlier while establishing the basicity criterion of the system of sines and cosines with linear phases in $L_{p}$. Thus, the following is valid.

Lemma 2.1. The following identities are true:

$$
\begin{aligned}
& \frac{1}{1-e^{i(\theta-\varphi)}}-\frac{1}{1-e^{i(\theta+\varphi)}}=-\frac{i}{2} \frac{\cos (\varphi / 2)}{\cos (\theta / 2)}\left[\frac{1}{\sin ((\varphi-\theta) / 2)}+\frac{1}{\sin ((\varphi+\theta) / 2)}\right] \\
& \frac{1}{1-e^{i(\theta-\varphi)}}-\frac{1}{1-e^{i(\theta+\varphi)}}=-\frac{i}{2} \frac{\sin (\varphi / 2)}{\sin (\theta / 2)}\left[\frac{1}{\sin ((\varphi-\theta) / 2)}-\frac{1}{\sin ((\varphi+\theta) / 2)}\right] .
\end{aligned}
$$


In the similar way, we obtain

$$
\begin{aligned}
& \frac{1}{1-e^{i(\theta-\varphi)}}+\frac{1}{1-e^{i(\theta+\varphi)}} \\
& \quad=\frac{e^{-i((\theta-\varphi) / 2)}}{e^{-i((\theta-\varphi) / 2)}-e^{i((\theta-\varphi) / 2)}}+\frac{e^{-i((\theta+\varphi) / 2)}}{e^{-i((\theta+\varphi) / 2)}-e^{i((\theta+\varphi) / 2)}} \\
& \quad=-\frac{1}{2 i} \frac{e^{-i((\theta-\varphi) / 2)}}{\sin ((\theta-\varphi) / 2)}-\frac{1}{2 i} \frac{e^{-i((\theta+\varphi) / 2)}}{\sin ((\theta+\varphi) / 2)} \\
& =-\frac{1}{2 i}\left[\frac{\cos ((\theta-\varphi) / 2)-i \sin ((\theta-\varphi) / 2)}{\sin ((\theta-\varphi) / 2)}+\frac{\cos ((\theta+\varphi) / 2)-i \sin ((\theta+\varphi) / 2)}{\sin ((\theta+\varphi) / 2)}\right] \\
& =-\frac{1}{2 i}\left[\frac{\cos ((\theta-\varphi) / 2)}{\sin ((\theta-\varphi) / 2)}+\frac{\cos ((\theta+\varphi) / 2)}{\sin ((\theta+\varphi) / 2)}\right]+1 \\
& =1-\frac{1}{2 i} \frac{\sin \theta}{\sin ((\theta-\varphi) / 2) \sin ((\theta+\varphi) / 2)} .
\end{aligned}
$$

Further, we must take into account the following relation:

$$
\begin{aligned}
& \frac{1}{\sin ((\theta-\varphi) / 2) \sin ((\theta+\varphi) / 2)}=\frac{1}{2 \sin (\theta / 2) \cos (\varphi / 2)}\left[\frac{1}{\sin ((\theta-\varphi) / 2)}+\frac{1}{\sin ((\theta+\varphi) / 2)}\right] \\
& \frac{1}{\sin ((\theta-\varphi) / 2) \sin ((\theta+\varphi) / 2)}=\frac{1}{2 \cos (\theta / 2) \sin (\varphi / 2)}\left[\frac{1}{\sin ((\theta-\varphi) / 2)}-\frac{1}{\sin ((\theta+\varphi) / 2)}\right] .
\end{aligned}
$$

As a result, we have

$$
\begin{aligned}
& e(\theta-\varphi)+e(\theta+\varphi)=1-\frac{1}{2 i} \frac{\sin (\theta / 2)}{\sin (\varphi / 2)}\left[\frac{1}{\sin ((\theta-\varphi) / 2)}-\frac{1}{\sin ((\theta+\varphi) / 2)}\right] \\
& e(\theta-\varphi)+e(\theta+\varphi)=1-\frac{1}{2 i} \frac{\cos (\theta / 2)}{\cos (\varphi / 2)}\left[\frac{1}{\sin ((\theta-\varphi) / 2)}+\frac{1}{\sin ((\theta+\varphi) / 2)}\right] .
\end{aligned}
$$

Assume

$$
K_{\varphi}(\theta)=e(\theta-\varphi)+e(\theta+\varphi)-1
$$

Thus,

$$
\begin{aligned}
K_{\varphi}(-\theta)= & -\frac{1}{2 i} \frac{\cos (\theta / 2)}{\cos (\varphi / 2)}\left[\frac{1}{-\sin ((\theta+\varphi) / 2)}-\frac{1}{\sin ((\theta-\varphi) / 2)}\right]=\frac{1}{2 i} \frac{\cos (\theta / 2)}{\cos (\varphi / 2)} \\
& \times\left[\frac{1}{\sin ((\theta+\varphi) / 2)}+\frac{1}{\sin ((\theta-\varphi) / 2)}\right]=-K_{\varphi}(\theta) .
\end{aligned}
$$

In sequel the following lemma is valid. 
Lemma 2.2. The following identities are true:

$$
\begin{aligned}
& \frac{1}{1-e^{i(\theta-\varphi)}}+\frac{1}{1-e^{i(\theta+\varphi)}}=1-\frac{1}{2 i} \frac{\sin (\theta / 2)}{\sin (\varphi / 2)}\left[\frac{1}{\sin ((\theta-\varphi) / 2)}-\frac{1}{\sin ((\theta+\varphi) / 2)}\right] \\
& \frac{1}{1-e^{i(\theta-\varphi)}}+\frac{1}{1-e^{i(\theta+\varphi)}}=1-\frac{1}{2 i} \frac{\cos (\theta / 2)}{\cos (\varphi / 2)}\left[\frac{1}{\sin ((\theta-\varphi) / 2)}-\frac{1}{\sin ((\theta+\varphi) / 2)}\right] .
\end{aligned}
$$

\section{Boundedness of Singular Operators on Subspace of Even Functions}

Let $f \in L_{p, \rho}^{-}$. We have

$$
\begin{aligned}
{[S f](t) } & =\frac{1}{2 \pi i} \int_{-\pi}^{\pi} \frac{f(s) d s}{1-e^{-i(s-t)}}=\frac{1}{2 \pi i}\left[\int_{0}^{\pi} \frac{f(s) d s}{1-e^{i(t-s)}}+\int_{-\pi}^{0} \frac{f(s) d s}{1-e^{i(t-s)}}\right] \\
& =\frac{1}{2 \pi i} \int_{0}^{\pi}\left[\frac{1}{1-e^{i(t-s)}}-\frac{1}{1-e^{i(t+s)}}\right] f(s) d s=-\frac{1}{4 \pi} \int_{0}^{\pi} K_{1}(t, s) f(s) d s \\
& =-\frac{1}{4 \pi} \int_{0}^{\pi} K_{2}(t, s) f(s) d s
\end{aligned}
$$

where the kernels $K_{i}(t, s), i=1,2$, are determined by the expressions

$$
\begin{aligned}
& K_{1}(t, s)=\frac{\cos (s / 2)}{\cos (t / 2)}\left[\frac{1}{\sin ((s-t) / 2)}+\frac{1}{\sin ((s+t) / 2)}\right], \\
& K_{2}(t, s)=\frac{\sin (s / 2)}{\sin (t / 2)}\left[\frac{1}{\sin ((s-t) / 2)}-\frac{1}{\sin ((s+t) / 2)}\right] .
\end{aligned}
$$

Continue the weight $\omega(t)$ to the interval $(-\pi, 0)$ by parity and denote by $\mu$ :

$$
\mu(t)= \begin{cases}\omega(t), & 0 \leq t \leq \pi \\ \omega(-t), & -\pi \leq t<0\end{cases}
$$

It is obvious that $\left\{ \pm \tau_{k}\right\}_{0}^{r} \subset[-\pi, \pi]$ are the degeneration points of $\mu(t)$. Thus, $\mu(t)=\omega(|t|)$, $t \in[-\pi, \pi]$, that is,

$$
\mu(t)=\prod_{k=0}^{r}|| t\left|-\tau_{k}\right|^{p \alpha_{k}}
$$

Accept the denotation

$$
f \sim g \text { as } t \longrightarrow a, \quad \text { if } \exists \delta>0: \delta \leq\left|\frac{f}{g}\right| \leq \delta^{-1}, \text { as } t \longrightarrow a \text {. }
$$


It is easy to see that the following holds

$$
|| t\left|-\tau_{k}\right| \sim\left|t-\tau_{k}\right|\left|t+\tau_{k}\right|, \quad t \in[-\pi, \pi], \text { for } k>1
$$

As a result, for $\mu$, we get the representation

$$
\mu(t) \sim \rho_{0}(t) \equiv|t|^{p \alpha_{0}} \prod_{k=1}^{r}\left|t-\tau_{k}\right|^{p \alpha_{k}}\left|t+\tau_{k}\right|^{p \alpha_{k}}, \quad t \in[-\pi, \pi] .
$$

It is obvious that the singular integral $S$ boundedly acts in $L_{p, \mu}(-\pi, \pi)$ if and only if it boundedly acts in $L_{p, \rho_{0}}(-\pi, \pi)$. Statement 1 is valid also in the case if the Cauchy kernel is replaced by the Hilbert kernel $(1 / \sin ((\varphi-t) / 2))$. So, assume that the inequalities $-1 / p<$ $\alpha_{k}<1 / q, k=\overline{0, r}$, are fulfilled. Then, from Statement 1 , we directly get that $S$ boundedly acts from $L_{p, \rho_{0}}^{-}$to $L_{p, \rho_{0}}$ and so from $L_{p, \mu}^{-}$to $L_{p, \mu}$. Assume

$$
k^{ \pm}(s, t)=\frac{\sin (s / 2)}{\sin (t / 2)} \frac{1}{\sin ((s \pm t) / 2)}, \quad(s, t) \in[-\pi, \pi] \times[-\pi, \pi],
$$

and consider the integral operator $I^{ \pm}$:

$$
I^{ \pm} f=\int_{-\pi}^{\pi} k^{ \pm}(s, t) f(s) d s
$$

with the kernel $k^{ \pm}(s, t)$. We have $\left(f \in L_{p, \mu}^{-}(-\pi, \pi)\right)$ :

$$
\|S f\|_{p, \mu}=\frac{1}{4 \pi}\left\|I^{-} \tilde{f}-I^{+} \tilde{f}\right\|_{p, \mu} \leq \frac{1}{4 \pi}\left(\left\|I^{-} \tilde{f}\right\|_{p, \mu}+\left\|I^{+} \tilde{f}\right\|_{p, \mu}\right),
$$

where

$$
\tilde{f}(t)= \begin{cases}f(t), & t \in[0, \pi], \\ 0, & t \in[-\pi, 0) .\end{cases}
$$

Consequently,

$$
\begin{aligned}
\left\|I^{-} \tilde{f}\right\|_{p, \mu}^{p} & =\int_{-\pi}^{\pi}\left|\left(I^{-} \tilde{f}\right)(t)\right|^{p} \mu(t) d t \sim \int_{-\pi}^{\pi}\left|I^{-} \tilde{f}\right|^{p} \rho_{0}(t) d t \\
& =\int_{-\pi}^{\pi}\left|\int_{-\pi}^{\pi} \frac{\tilde{f}(s) \sin (s / 2) d s}{\sin ((s-t) / 2)}\right|^{p} \rho_{0}(t)\left|\sin \frac{t}{2}\right|^{-p} d t .
\end{aligned}
$$

It is obvious that

$$
\rho_{0}(t)\left|\sin \frac{t}{2}\right|^{-p} \sim \theta(t)
$$


where $\theta(t)=|s|^{p\left(\alpha_{0}-1\right)} \prod_{k=1}^{r}\left|t-\tau_{k}\right|^{p \alpha_{k}}\left|t+\tau_{k}\right|^{p \alpha_{k}}$. So, if the inequalities

$$
-\frac{1}{p}<\alpha_{0}-1<\frac{1}{q}, \quad-\frac{1}{p}<\alpha_{k}<\frac{1}{q}, \quad k=\overline{1, r}
$$

hold, then from Statement 1 we obtain

$$
\begin{aligned}
\left\|I^{-} \tilde{f}\right\|_{L_{p, \mu}}^{p} & \leq c \int_{-\pi}^{\pi}\left|\int_{-\pi}^{\pi} \frac{\tilde{f}(s) \sin (s / 2)}{\sin ((s-t) / 2)} d s\right|^{p} \theta(t) d t \leq c\left\|\tilde{f}(s) \sin \frac{s \theta(s)}{2}\right\|_{L_{p, \theta}}^{p} \\
& \leq c \int_{0}^{\pi}|f(s) s|^{p} \theta(s) d s=c\|f\|_{L_{p, \omega^{\prime}}^{0}}^{p}
\end{aligned}
$$

where $c$ is a constant independent from $f$ (different in different places). As a result, we get that if the inequalities (3.14) hold, the operator $I^{-}$boundedly acts from $L_{p, \omega}^{0}$ to $L_{p, \mu}$. The same conclusion is true for the operator $I^{+}$as well. As a result, we get that while fulfilling the conditions

$$
\alpha_{0} \in\left(-\frac{1}{p}, \frac{1}{q}\right) \bigcup\left(\frac{1}{q}, 1+\frac{1}{q}\right), \quad \alpha_{k} \in\left(-\frac{1}{p}, \frac{1}{q}\right), \quad k=\overline{1, r}
$$

the operator $S$ boundedly acts from $L_{p, \mu}^{-}$to $L_{p, \mu}$. On the other hand, it is easily seen that $[S f](-t)=[S f](t)$. As a result, we get that the operator $S$ boundedly acts from $L_{p, \mu}^{-}$to $L_{p, \mu}^{+}$.

Now, consider the case when $\alpha_{0}=1 / q$ and $\alpha_{k}, k=\overline{1, r}$, satisfy conditions (3.16). Let $p_{ \pm \varepsilon}=p \pm \varepsilon$ and $q_{ \pm \varepsilon}$ be a number conjugated to $p_{ \pm \varepsilon}$. It is obvious that the relations

$$
\alpha_{0} \in\left(-\frac{1}{p_{ \pm \varepsilon}}, \frac{1}{q_{ \pm \varepsilon}}\right) \bigcup\left(\frac{1}{q_{ \pm \varepsilon}}, 1+\frac{1}{q_{ \pm \varepsilon}}\right), \quad \alpha_{k} \in\left(-\frac{1}{p_{ \pm \varepsilon}}, \frac{1}{q_{ \pm \varepsilon}}\right), \quad k=\overline{1, r},
$$

are fulfilled for sufficiently small $\varepsilon>0$. Then, from the previous reasonings we get that the operator $S$ boundedly acts from $L_{p_{ \pm \varepsilon}, \mu}^{-}$to $L_{p_{ \pm \varepsilon}, \mu}^{+}$. As a result, it follows from the Riesz-Torin theorem (see, e.g., [7, page 144]) that the operator $S$ boundedly acts from $L_{p, \mu}^{-}$to $L_{p, \mu}^{+}$. We get the following.

Statement 2. Let the inequalities

$$
-\frac{1}{p}<\alpha_{0}<2-\frac{1}{p}, \quad-\frac{1}{p}<\alpha_{k}<\frac{1}{q}, \quad k=\overline{1, r}
$$

be fulfilled. Then, the operator $S$ boundedly acts from $L_{p, \mu}^{-}$to $L_{p, \mu}^{+}$.

Now, consider the representation of the operator $S$ by the kernel $K_{1}(t, s)$. Having paid attention to the expression

$$
\frac{\cos (s / 2)}{\cos (t / 2)}=\frac{\sin ((\pi-s) / 2)}{\sin ((\pi-t) / 2)}
$$


similar to the previous case we establish that the boundedness of the operator $S$ holds also in the case when the change interval of the exponent $\alpha_{r}$ extends by $(-1 / p, 2-1 / p)$. In the conclusion we get that the following main theorem is valid.

Theorem 3.1. Let the weight function $\omega$ be defined by the expression (2.1) and assume that the inequalities

$$
-\frac{1}{p}<\alpha_{0}<2-\frac{1}{p^{\prime}}, \quad-\frac{1}{p}<\alpha_{m}<2-\frac{1}{p^{\prime}}, \quad-\frac{1}{p}<\alpha_{k}<\frac{1}{q}, \quad k=\overline{1, r-1}
$$

are fulfilled. Then the singular operator S:

$$
S f=\int_{-\pi}^{\pi} k(t, s) f(s) d s
$$

with Cauchy kernel $k(t, s)=1 /\left(1-e^{-i(s-t)}\right)$, boundedly acts from $L_{p, \mu}^{-}$to $L_{p, \mu^{\prime}}^{+}$where $\mu(t)=\omega(|t|)$, $t \in(-\pi, \pi)$.

\section{Boundedness of Singular Operators on Subspace of Odd Functions}

Let the weight function $\omega(t)$ be defined by expression (2.1) and assume $\mu(t) \equiv \omega(|t|), t \in$ $(-\pi, \pi)$. Denote by $K(s ; t)$ the following Cauchy-type kernel

$$
K(s ; t)=\frac{1}{1-e^{i(t-s)}}-\frac{1}{2}, \quad(s ; t) \in(-\pi, \pi) \times(-\pi, \pi) .
$$

Appropriate integral operator denote by $\mathcal{~}$ :

$$
[\mathcal{K} f](t)=\frac{1}{2 \pi} \int_{-\pi}^{\pi} K(s ; t) f(s) d s
$$

Let the following inequalities

$$
-\frac{1}{p}<\alpha_{k}<\frac{1}{q}, \quad k=\overline{0, r}
$$

be fulfilled. We have

$$
\int_{-\pi}^{\pi}|f(t)| d t=\int_{-\pi}^{\pi}|f(t)| \mu^{1 / p} \mu^{-1 / p} d t \leq\|f\|_{p, \mu}\left(\int_{-\pi}^{\pi} \mu^{-q / p} d t\right)^{1 / q}
$$

From (4.3) it follows that $-q \alpha_{k}>-1, k=\overline{0, r}$, and as a result $\mu^{-q / p} \in L_{1}(-\pi, \pi)$. As a result, from Statement 1 we obtain that the integral operator $\mathcal{K}$ boundedly acts in $L_{p, \mu}$, if the 
inequalities (4.3) hold. In particular, it follows that the operator $\mathcal{K}$ boundedly acts from $L_{p, \mu}^{+}$ to $L_{p, \mu}$ if the inequalities (4.3) are fulfilled, that is,

$$
\|\mathcal{K} f\|_{L_{p, \mu}} \leq c\|f\|_{L_{p, \mu^{\prime}}^{+}} \quad \forall f \in L_{p, \mu^{\prime}}^{+}
$$

where $c$ is a constant independent from $f$. On the other hand for $f \in L_{p, \mu}^{+}$we have

$$
[\mathcal{K} f](t)=\frac{1}{2 \pi} \int_{0}^{\pi} f(s)[K(s ; t)+K(-s ; t)-1] d s .
$$

Pay an attention to the relation (2.13), we obtain that $[\mathcal{K} f](-t)=-[\mathcal{K} f](t), t \in(-\pi, \pi)$. Then from (4.5) yields

$$
\|\mathcal{K} f\|_{L_{p, \omega}^{0}} \leq c\|f\|_{L_{p, \omega}^{0},} \quad \forall f \in L_{p, \omega}^{0} .
$$

Now, let

$$
-1-\frac{1}{p}<\alpha_{0}<-\frac{1}{p}, \quad-\frac{1}{p}<\alpha_{k}<\frac{1}{q}, \quad k=\overline{1, r}
$$

be fulfilled. Assume

$$
K^{ \pm}(s ; t)=-\frac{1}{2 i} \frac{\sin (t / 2)}{\sin (s / 2) \sin ((t \mp s) / 2)}, \quad(s ; t) \in(0, \pi) \times(0, \pi) .
$$

Denote the integral operator with kernel $K^{ \pm}(s ; t)$ by $S^{ \pm}$, that is,

$$
\left[S^{ \pm} f\right](t)=\frac{1}{2 \pi} \int_{0}^{\pi} K^{ \pm}(s ; t) f(s) d s, \quad t \in(0, \pi) .
$$

Taking into account the relation (2.10), for $f \in L_{p, \mu}^{+}$we have

$$
\begin{aligned}
{[\mathcal{K} f](t) } & =\frac{1}{2 \pi} \int_{0}^{\pi} f(s)[K(s ; t)+K(-s ; t)-1] d s \\
& =\frac{1}{2 \pi} \int_{0}^{\pi}\left[K^{+}(s ; t)-K^{-}(s ; t)\right] f(s) d s=\left[S^{+}-S^{-}\right] f
\end{aligned}
$$

that is,

$$
[\mathcal{K} f](t)=\left[\left(S^{+}-S^{-}\right) f\right](t), \quad t \in(-\pi, \pi) .
$$

Take into account the nonparity $[\mathcal{K} f](t)$ on $(-\pi, \pi)$ we obtain

$$
2\|\mathcal{K} f\|_{L_{p, \omega}^{0}}^{p}=\|\mathcal{K} f\|_{L_{p, \mu}}^{p} \leq\left(\left\|S^{+} f\right\|_{L_{p, \mu}}+\left\|S^{-} f\right\|_{L_{p, \mu}}\right)^{p} .
$$


Let

$$
\tilde{f}(t)= \begin{cases}f(t), & t \in(0, \pi), \\ 0, & t \in(-\pi, 0) .\end{cases}
$$

Consider the operator $S^{+}$. We have

$$
\left[S^{+} f\right](t)=\frac{\sin (t / 2)}{2 \pi} \int_{0}^{\pi} \frac{f(s) \sin ^{-1}(s / 2)}{\sin ((t-s) / 2)} d s=\frac{\sin (t / 2)}{2 \pi} \int_{-\pi}^{\pi} \frac{\tilde{f}(s) \sin ^{-1}(s / 2)}{\sin ((t-s) / 2)} d s
$$

Thus

$$
\left\|S^{+} f\right\|_{L_{p, t}}^{p}=c \int_{-\pi}^{\pi}\left|\left[S^{+} f\right](t)\right|^{p} \mu(t) d t=c \int_{-\pi}^{\pi}\left|\int_{-\pi}^{\pi} \frac{\tilde{f}(s) \sin ^{-1}(s / 2)}{\sin ((t-s) / 2)} d s\right|^{p}\left|\sin \frac{t}{2}\right|^{p} \mu(t) d t .
$$

Further, we must take into account the expression $\sin (t / 2) \sim t, t \in(-\pi, \pi)$. As a result, from the previous relation we have

$$
\left\|S^{+} f\right\|_{L_{p, \mu}}^{p} \leq c \int_{-\pi}^{\pi}\left|\int_{-\pi}^{\pi} \frac{\tilde{f}(s) \sin ^{-1}(s / 2)}{\sin ((t-s) / 2)} d s\right|^{p} \tilde{\mu}(t) d t
$$

where

$$
\tilde{\mu}(t)=|t|^{p\left(\alpha_{0}+1\right)} \prod_{k=1}^{r}|| t\left|-t_{k}\right|^{p \alpha_{k}}
$$

It is clear that for weight function $\tilde{\mu}(t)$ Muckenhoupt condition is fulfilled and applying Statement 1 to the expression (4.17) we obtain

$$
\begin{aligned}
\left\|S^{+} f\right\|_{L_{p, \mu}}^{p} & \leq c\left\|\tilde{f}(s) \sin ^{-1} \frac{s}{2}\right\|_{L_{p, \tilde{\mu}}}^{p}=c \int_{-\pi}^{\pi}|\tilde{f}(s)|^{p}\left|\sin ^{-1} \frac{s}{2}\right|^{p} \tilde{\mu}(s) d s \\
& \leq c \int_{0}^{\pi}|f(s)|^{p}|s|^{-p} \tilde{\mu}(s) d s=c \int_{0}^{\pi}|f(s)|^{p} \omega(s) d s=c\|f\|_{L_{p, \mu}^{+}}^{p} .
\end{aligned}
$$

In the similar way we establish the validity of the inequality

$$
\left\|S^{-} f\right\|_{L_{p, \mu}} \leq c\|f\|_{L_{p, \mu^{\prime}}^{+}} \quad \forall f \in L_{p, \mu}^{+} .
$$

If the inequalities (4.8) hold, as a result, we have

$$
\|\mathcal{K} f\|_{L_{p, \mu}} \leq c\|f\|_{L_{p, \mu^{\prime}}^{+}} \quad \forall f \in L_{p, \mu^{\prime}}^{+} .
$$


Consider the case

$$
\alpha_{0}=-\frac{1}{p^{\prime}}, \quad-\frac{1}{p}<\alpha_{k}<\frac{1}{q}, \quad k=\overline{1, r}
$$

Take sufficiently small $\varepsilon>0$ and determine $p_{\varepsilon}^{ \pm}=p \pm \varepsilon$. Acting similarly to the case $L_{p, \mu}^{-}$(par.3) and accept the Riesz-Torin theorem we obtain boundedly acting of the operator $\mathcal{K}$ from $L_{p, \mu}^{+}$ to $L_{p, \mu}^{-}$(since $\left.[\mathcal{K} f](t) \in L_{p, \mu}^{-}\right)$. Thus, if the following inequalities

$$
-1-\frac{1}{p}<\alpha_{0}<\frac{1}{q}, \quad-\frac{1}{p}<\alpha_{k}<\frac{1}{q}, \quad k=\overline{1, r},
$$

are fulfilled, then the operator $\mathcal{K}$ boundedly acts from $L_{p, \mu}^{+}$to $L_{p, \mu}^{-}$.

Using the identity (2.11) in the similar way we establish that the same conclusion with respect to the operator $\mathcal{K}$ is true in the case when the change interval of the exponent $\alpha_{r}$ is expanded on $(-1-1 / p, 1 / q)$. As a result, we obtain the validity of the following theorem.

Theorem 4.1. Let the weight function $\omega$ be defined by the expression (2.1) and $\mu(t) \equiv \omega(|t|), t \in$ $(-\pi, \pi)$. Assume that the inequalities

$$
-1-\frac{1}{p}<\alpha_{0}<\frac{1}{q}, \quad-1-\frac{1}{p}<\alpha_{r}<\frac{1}{q}, \quad-\frac{1}{p}<\alpha_{k}<\frac{1}{q}, \quad k=\overline{1, r-1},
$$

are fulfilled. Then the singular operator $\boldsymbol{\alpha}$ :

$$
\mathcal{K} f=\int_{-\pi}^{\pi} K(s ; t) f(s) d s
$$

with Cauchy-type kernel $K(s ; t)=\left(1 /\left(1-e^{-i(s-t)}\right)\right)-1 / 2$, boundedly acts from $L_{p, \mu}^{+}$to $L_{p, \mu}^{-}$.

\section{Completeness, Minimality, and Basicity of the System of Sines in Weight Space}

Consider the system of sines $\{\sin n t\}_{n \in N}$. Let conditions (3.20) be fulfilled. It is easy to see that then the system $\{\sin n t\}_{n \in N}$ is minimal in $L_{p, \omega}^{0}$. The system $\left\{(2 / \pi) \omega^{-1}(t) \sin n t\right\}_{n \in N}, 1 / p+$ $1 / q=1$, is a biorthogonal system to it. Indeed, it is obvious that $L_{q, \omega}^{0}$ is a space conjugated to $L_{p, \omega}^{0}$, and an arbitrary continuous functional $l_{g}$ on $L_{p, \omega}^{0}$, generated by $g \in L_{q, \omega}^{0}$, realized by the formula

$$
l_{g}(f)=\int_{0}^{\pi} f \bar{g} \omega d t, \quad \forall f \in L_{p, \omega}^{0}
$$

where $(\cdot)$ is a complex conjugation. 
Take $g(t)=\omega^{-1}(t) \sin n t, n \in N$. We have

$$
\left\|\omega^{-1}(t) \sin n t\right\|_{L_{q, \omega}^{0}}^{q}=\int_{0}^{\pi}\left|\omega^{-1}(t) \sin n t\right|^{q} \omega(t) d t=\int_{0}^{\pi} \omega^{1-q}(t)|\sin n t|^{q} d t
$$

Since $\sin n t \sim t$ as $t \rightarrow 0$ and $\sin n t \sim \pi-t$ as $t \rightarrow \pi$ for every fixed $n \in N$, then from relation (5.2) follows that $\left\{\omega^{-1} \sin n t\right\}_{n \in N} \subset L_{q, \omega}^{0}$, if the inequalities

$$
\alpha_{0}<2-\frac{1}{p^{\prime}}, \quad \alpha_{m}<2-\frac{1}{p}, \quad \alpha_{k}<\frac{1}{q}, \quad k=\overline{1, r-1},
$$

are fulfilled.

Take $g(t)=(2 / \pi) \omega^{-1}(t) \sin n t$ and denote by $\vartheta_{n}$ generated by its functional, that is,

$$
\vartheta_{n}(f)=\frac{2}{\pi} \int_{0}^{\pi} f(t) \sin n t d t, \quad \forall f \in L_{p, \omega}^{0}
$$

It is clear that $\vartheta_{n}(\sin k t)=\delta_{n k}, \forall n, k \in N$, where $\delta_{n k}$ is a Kronecker's symbol. Consider

$$
\|\sin n t\|_{L_{p, \omega}^{0}}^{p}=\int_{0}^{\pi}|\sin n t|^{p} \omega(t) d t
$$

Thus, $\{\sin n t\}_{n \in N} \subset L_{p, \omega}$, if $\alpha_{0}>-1 / p-1, \alpha_{r}>-1 / p-1, \alpha_{k}>-1 / p, k=\overline{0, r-1}$. As a result, we obtain that if the inequalities

$$
-1-\frac{1}{p}<\alpha_{0}<2-\frac{1}{p^{\prime}}, \quad-1-\frac{1}{p}<\alpha_{r}<2-\frac{1}{p^{\prime}}, \quad-\frac{1}{p}<\alpha_{k}<\frac{1}{q}, \quad k=\overline{0, r-1},
$$

hold, then the system $\{\sin n t\}_{n \in N}$ is minimal in $L_{p, \omega}^{0}$.

Now consider the completeness of the system $\{\sin n t\}_{n \in N}$ in $L_{p, \omega}^{0}$. Suppose that for some $g \in L_{q, \omega}^{0}$,

$$
\int_{0}^{\pi} \sin n t \bar{g} \omega d t=0, \quad \forall n \in N,
$$

holds. We have

$$
\int_{0}^{\pi}|\bar{g} \omega| d t=\int_{0}^{\pi}|\bar{g}| \omega^{1 / q} \omega^{1 / p} d t \leq\|g\|_{L_{q, \omega}^{0}}\left(\int_{0}^{\pi} \omega d t\right)^{1 / p} .
$$

It is easy to see that if the inequalities

$$
\alpha_{k}>-\frac{1}{p}, \quad k=\overline{0, r}
$$


are fulfilled, then $\omega \in L_{1}$. Then from the previous relation we get $\bar{g} \omega \in L_{1}$. Since, the system $\{\sin n t\}_{n \in N}$ is complete in space of continuous on $[0, \pi]$ functions with sup-norm, which vanishes at the ends of the segment $[0, \pi]$, then from (5.7) it follows that $g(t)=0$ a.e. on $(0, \pi)$. Consequently, the system $\{\sin n t\}_{n \in N}$ is complete in $L_{p, \omega}^{0}$. So, the following statement is true.

Statement 3. Let the weight function $\omega(t)$ be defined by expression (2.1). The system of sines $\{\sin n t\}_{n \in N}$ is minimal in $L_{p, \omega}^{0}$, if the inequalities (5.6) are fulfilled. It is complete in $L_{p, \omega}^{0}$, if the inequalities (5.9) are fulfilled. Moreover, it forms a basis in $L_{p, \omega}^{0}$, if the inequalities

$$
-\frac{1}{p}<\alpha_{k}<\frac{1}{q}, \quad k=\overline{0, m}
$$

hold.

The basicity of system of sines in $L_{p, \omega}^{0}$, when the inequalities (5.10) hold, follows from the basicity of system of exponent $\left\{e^{i n t}\right\}_{n \in Z}$ in $L_{p, \mu}$, where $\mu(t)=\omega(|t|), t \in(-\pi, \pi)$. The basicity of these systems earlier considered in papers $[3,4,8,9]$.

In the similar way we prove the following statement.

Statement 4. Let the weight function $\omega(t)$ defined by expression (2.1). The system of cosines $1 \cup\{\cos n t\}_{n \in N}$ is minimal (forms a basis) in $L_{p, \omega}^{0}$, if the inequalities (5.10) are fulfilled. It is complete in $L_{p, \omega}^{0}$, if the inequalities (5.9) holds.

\section{References}

[1] I. I. Danilyuk, Irregular Boundary Value Problems on a Plane, Nauka, Moscow, Russia, 1975.

[2] B. S. Pavlov, "Basicity of an exponential system and Muckenhoupt's condition," Doklady Akademii Nauk SSSR, vol. 247, no. 1, pp. 37-40, 1979 (Russian).

[3] K. S. Kazaryan and P. I. Lizorkin, "Multipliers, bases and unconditional bases of the weighted spaces B and SB," Akademiya Nauk SSSR. Trudy Matematicheskogo Instituta imeni V. A. Steklova, vol. 187, pp. 111-130, 1989.

[4] S. S. Pukhov and A. M. Sedletskiy, "Bases of exponents, sines and cosines in weight spaces on finite interval," Proceedings of the Russian Academy of Sciences, vol. 425, no. 4, pp. 452-455, 2009.

[5] E. I. Moiseev, "The basis property for systems of sines and cosines," Doklady Akademii Nauk SSSR, vol. 275, no. 4, pp. 794-798, 1984.

[6] E. I. Moiseev, "On the basis property of a system of sines," Differentsial'nie Uravnenia, vol. 23, no. 1, pp. 177-179, 1987.

[7] G. G. Devdariani, “The basis property of a system of functions," Differentsial'nie Uravnenia, vol. 22, no. 1, pp. 170-171, 1986.

[8] E. I. Moiseev, "On basicity of system of sines and cosines in weight space," Differentsial'nie Uravnenia, vol. 34, no. 1, pp. 40-44, 1998.

[9] B. T. Bilalov and S. G. Veliev, "Bases from the eigenfunctions of two discountinuous differential operators," Differentsial'nie Uravnenia, vol. 42, no. 9, pp. 190-192, 2006. 


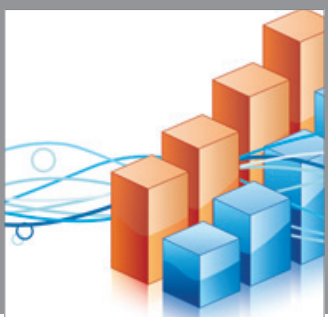

Advances in

Operations Research

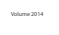

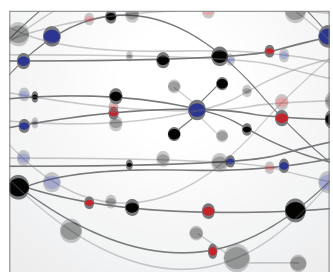

\section{The Scientific} World Journal
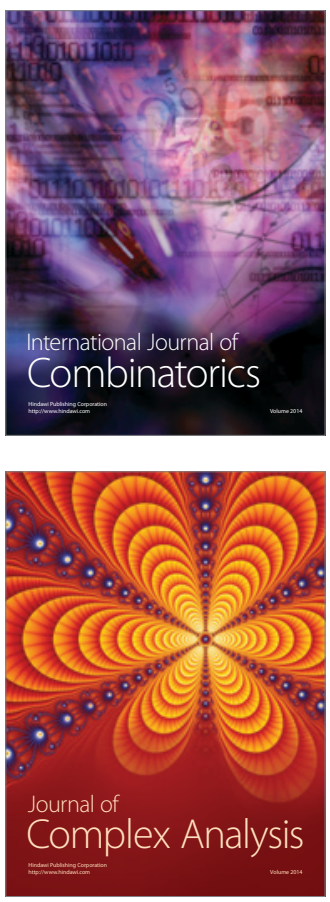

International Journal of

Mathematics and

Mathematical

Sciences
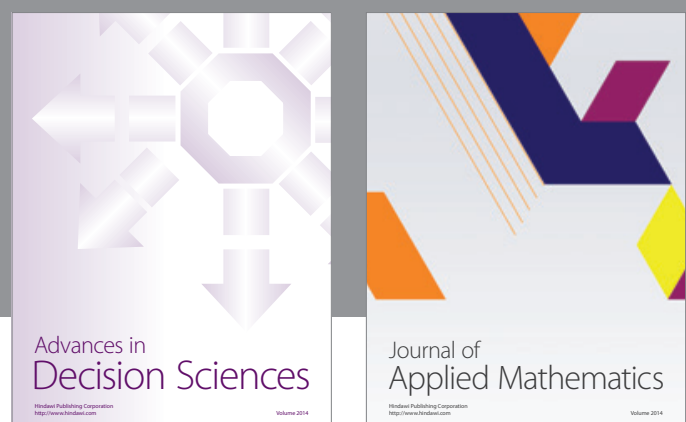

Journal of

Applied Mathematics
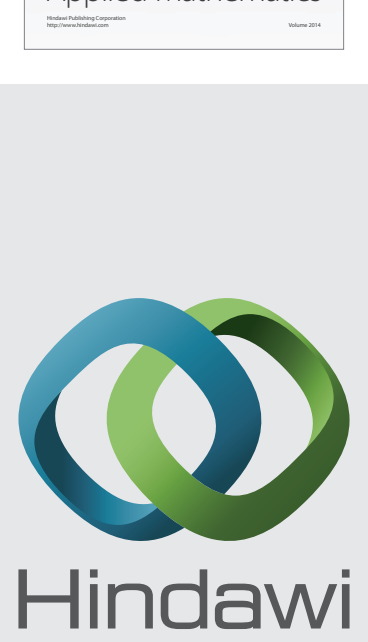

Submit your manuscripts at http://www.hindawi.com
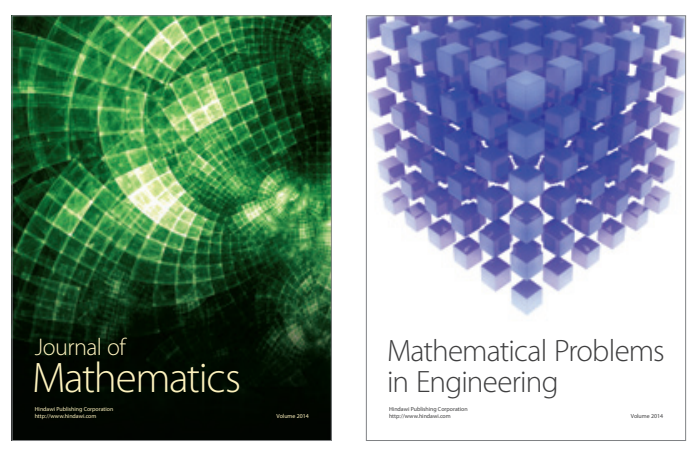

Mathematical Problems in Engineering
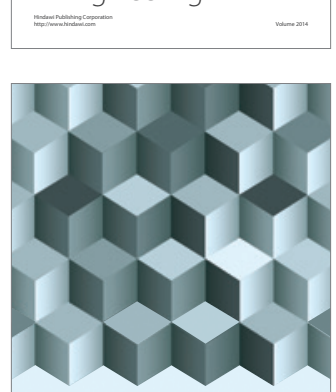

Journal of

Function Spaces
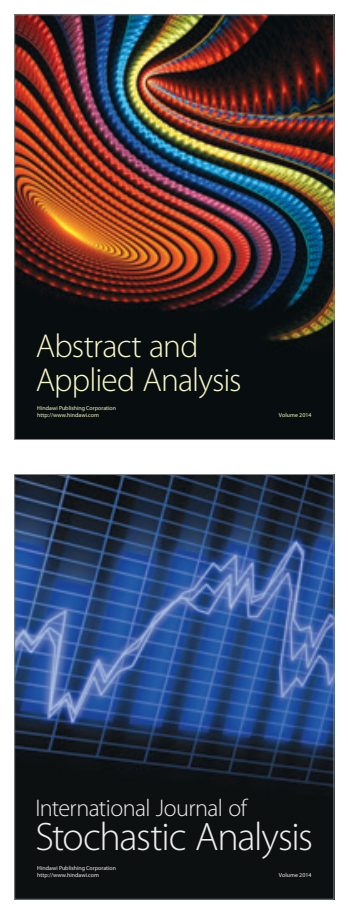

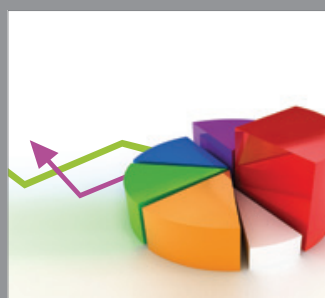

ournal of

Probability and Statistics

Promensencen
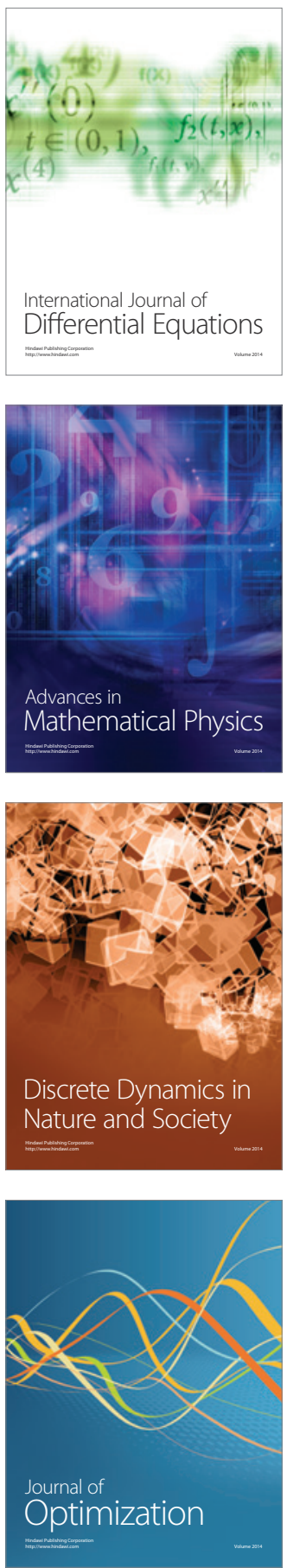